\title{
Practicum Direct
}

Alejandro Puente-Castro, Brais Galdo, Juan R Rabuñal, Alejandro Pazos

The current SARS-CoV-2 pandemic has put the entire civilization, particularly medical systems around the world, to the test. Managers and decision-makers in the health-care system must maximize resource management. Because of their predictive capacity, Artificial Intelligence (AI) tools and procedures are extremely useful in decision-making in emergency situations including severe pandemics. The PRACTICUM DIRECT project is presented in this paper, which proposes the design and development of a tool to aid health system managers in making early management decisions of hospital resources using $\mathrm{Al}$ approaches. 